\title{
Formulations of Solutions for Earthquake Source Models and Some Related Problems
}

\author{
By \\ Ryosuke Sato \\ Geophysical Institute, Faculty of Science, \\ University of Tokyo
}

\begin{abstract}
Expressions for displacement potentials due to a dip-slip fault and a strike-slip fault model with arbitrary dip angle are presented in Cartesian coordinates, cylindrical coordinates and spherical coordinates. From these results, displacements and stresses when the time variation of the source is a step function type are also derived.

It is shown that the solutions obtained above can easily be expanded to those for moving dislocation models.

In the last part of this paper, the solutions in polar coordinates are expressed in another polar coordinates with different origin. The expressions can immediately be applied to the problems of generation of seismic waves and excitation of the free oscillations of the earth due to a dislocation in a spherical earth.
\end{abstract}

\section{§1. Introduction}

There are many works which give the mathematical expressions of displacements due to earthquake source models. Many of them, however, are lengthy and difficult to understand.

In the present paper, compact and simple expressions for a strike-slip fault and for a dip-slip fault are presented with some applications.

\section{§ 2. Single force case}

The displacement components, $u_{x}, u_{y}, u_{z}$, due to a single force of magnitude $\chi(t)$ acting at the origin in the direction of the $x$-axis given by Love (1927) are

$$
\begin{aligned}
& u_{x}=\frac{1}{4 \pi \rho}\left[\frac{\partial^{2} r^{-1}}{\partial x^{2}} \int_{r / v_{p}}^{r / v_{a}} t^{\prime} \chi\left(t-t^{\prime}\right) d t^{\prime}+\frac{1}{r}\left(\frac{\partial r}{\partial x}\right)^{2}\left\{\frac{1}{v_{p}^{2}} \chi\left(t-\frac{r}{v_{p}}\right)-\frac{1}{v_{a}^{2}} \chi\left(t-\frac{r}{v_{s}}\right)\right\}+\frac{1}{v_{s}^{2} r} \chi\left(t-\frac{r}{v_{s}}\right)\right], \\
& u_{u}=\frac{1}{4 \pi \rho}\left[\frac{\partial^{2} r^{-1}}{\partial x \partial y} \int_{r / v_{p}}^{r / v_{s}} t^{\prime} \chi\left(t-t^{\prime}\right) d t^{\prime}+\frac{1}{r} \frac{\partial r}{\partial x} \frac{\partial r}{\partial y}\left\{\frac{1}{v_{p}^{2}} \chi\left(t-\frac{r}{v_{p}}\right)-\frac{1}{v_{s}^{2}} \chi\left(t-\frac{r}{v_{s}}\right)\right\}\right], \\
& u_{x}=\frac{1}{4 \pi \rho}\left[\frac{\partial^{2} r^{-1}}{\partial x \partial z} \int_{r / v_{p}}^{r / v_{a}} t^{\prime} \chi\left(t-t^{\prime}\right) d t^{\prime}+\frac{1}{r} \frac{\partial r}{\partial x} \frac{\partial r}{\partial z}\left\{\frac{1}{v_{p}^{2}} \chi\left(t-\frac{r}{v_{p}}\right)-\frac{1}{v_{a}^{2}} \chi\left(t-\frac{r}{v_{s}}\right)\right\}\right],
\end{aligned}
$$

where $v_{p}$ and $v_{s}$ are the dilatational and the shear velocities, respectively, and

$$
r=\sqrt{x^{2}+y^{2}+z^{2}} \text {. }
$$

According to Keilis-Borok (1950), by introducing new functions, defined by

$$
\left.\begin{array}{rl}
\phi & =\frac{1}{r} F\left(t-\frac{r}{v_{p}}\right), \\
\phi & =\frac{1}{r} F\left(t-\frac{r}{v_{s}}\right), \\
F(t) & =\int_{0}^{t} d s^{\prime} \int_{0}^{g^{\prime}} \chi(s) d s,
\end{array}\right\}
$$

(1) is reduced to

$$
\left.\begin{array}{l}
u_{x}=\frac{1}{4 \pi \rho}\left\{\frac{\partial^{2}}{\partial x^{2}}(\phi-\phi)+\nabla^{2} \phi\right\}, \\
u_{y}=\frac{1}{4 \pi \rho} \frac{\partial^{2}}{\partial x \partial y}(\phi-\phi), \\
u_{z}=\frac{1}{4 \pi \rho} \frac{\partial^{2}}{\partial x \partial z}(\phi-\phi),
\end{array}\right\}
$$

which can be written in a compact vectorial form as

with

$$
\boldsymbol{u}=\operatorname{grad} \Phi+\operatorname{curl} \operatorname{curl}\left(\Psi_{1}, 0,0\right)
$$

$$
\Phi=\frac{1}{4 \pi \rho} \frac{\partial \phi}{\partial x}, \quad \Psi_{1}=-\frac{1}{4 \pi \rho} \psi
$$


If the force is directed along the $y$-axis, we have

with

$$
\boldsymbol{u}=\operatorname{grad} \Phi+\operatorname{curl} \operatorname{curl}\left(0, \Psi_{2}, 0\right)
$$

$$
\Phi=\frac{1}{4 \pi \rho} \frac{\partial \phi}{\partial y}, \quad \Psi_{2}=-\frac{1}{4 \pi \rho} \psi .
$$

A similar expression may be easily found when the force is directed along the $z$-axis.

If $\chi(t)=\chi_{0} e^{i \omega t}$, then

$$
\left.\begin{array}{l}
\phi=-\frac{\chi_{0}}{\omega^{2} r} e^{i \omega\left(t-r / v_{p}\right)}=\frac{i \chi_{0}}{\omega v_{p}} h_{0}^{(2)}\left(\frac{\omega}{v_{p}} r\right) e^{i \omega t}, \\
\phi=\frac{i \chi_{0}}{\omega v_{s}} h_{0}^{(2)}\left(\frac{\omega}{v_{s}} r\right) e^{i \omega t},
\end{array}\right\}
$$

where $h_{0}{ }^{(2)}(z)$ is the spherical Hankel function of the second kind. $\chi_{0}(\omega)$ should be understood as the Fourier transform of $\chi(t)$;

$$
\chi_{0}(\omega)=\int_{-\infty}^{\infty} \chi(t) e^{-i \omega t} d t .
$$

Therefore if we apply an operator

$$
\frac{1}{2 \pi} \int_{-\infty}^{\infty} d \omega
$$

to the solution when $\phi$ and $\phi$ are given by (8), we can immediately obtain the solution for an arbitrary time variation $\chi(t)$.

\section{§3. Single couple case}

Displacements due to a force system of double forces with moment, as shown in Fig. 1, can be obtained from

with

$$
\boldsymbol{u}=\operatorname{grad} \Phi+\operatorname{curl} \operatorname{curl}\left(\Psi_{1}, 0,0\right)
$$

$$
\Phi=-\frac{1}{4 \pi \rho} \frac{\partial^{2} \phi}{\partial x \partial y}, \quad \Psi_{1}=\frac{1}{4 \pi \rho} \frac{\partial \phi}{\partial y},
$$

where, in the definition of $\phi$ and $\phi$ in (2) or $(8)$, the moment, $M(t)$, should be taken in place of $\chi(t)$.

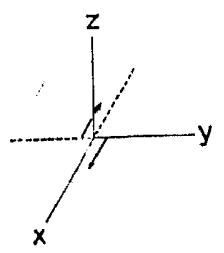

Fig. 1.

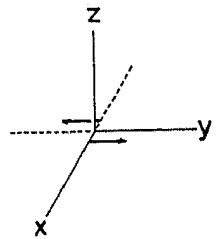

Fig. 2.

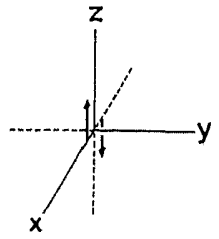

Fig. 3.
When one of the forces is directed along the $y$-axis, as shown in Fig. 2, the displacement vector is

$$
\boldsymbol{u}=\operatorname{grad} \Phi+\operatorname{curl} \operatorname{curl}\left(0, \Psi_{2}, 0\right)
$$

with

$$
\Phi=-\frac{1}{4 \pi \rho} \frac{\partial^{2} \phi}{\partial x \partial y}, \quad \Psi_{2}=\frac{1}{4 \pi \rho} \frac{\partial \psi}{\partial x} .
$$

Thus we can find the displacement expressions for any single couple orientation. For example, for the force system, as shown in Fig. 3, we have

$$
\boldsymbol{u}=\operatorname{grad} \Phi+\operatorname{curl} \operatorname{curl}\left(0,0, \Psi_{\mathbf{3}}\right)
$$

with

$$
\Phi=-\frac{1}{4 \pi \rho} \frac{\partial^{2} \phi}{\partial z \partial x}, \quad \Psi_{3}=\frac{1}{4 \pi \rho} \frac{\partial \psi}{\partial \dot{x}} .
$$

\section{§4. Double couple case}

The double couple in Fig. 4 is given by the superposition of (10) and (12). Hence

$$
\boldsymbol{u}=\operatorname{grad} \Phi+\operatorname{curl} \operatorname{curl}\left(\Psi_{1}, \Psi_{2}, 0\right)
$$

with

$$
\left.\begin{array}{rl}
\Phi & =-\frac{1}{2 \pi \rho} \frac{\partial^{2} \phi}{\partial x \partial y}, \\
\Psi_{1} & =\frac{1}{4 \pi \rho} \frac{\partial \psi}{\partial y}, \quad \Psi_{2}=\frac{1}{4 \pi \rho} \frac{\partial \psi}{\partial x} .
\end{array}\right\}
$$

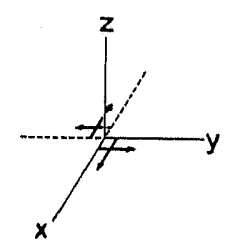

Fig. 4.

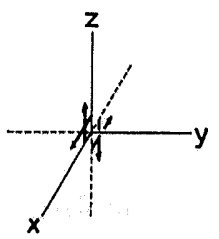

Fig. 5.
When the double couple lies in the $x z$-plane, as illustrated in Fig. 5, we have

$$
\boldsymbol{u}=\operatorname{grad} \Phi+\operatorname{curl} \operatorname{curl}\left(\Psi_{1}, 0, \Psi_{3}\right)
$$

with

$$
\left.\begin{array}{rl}
\Phi & =-\frac{1}{2 \pi \rho} \frac{\partial^{2} \phi}{\partial z \partial x}, \\
\Psi_{1} & =\frac{1}{4 \pi \rho} \frac{\partial \psi}{\partial z}, \quad \Psi_{s}=\frac{1}{4 \pi \rho} \frac{\partial \psi}{\partial x} .
\end{array}\right\}
$$

Thus we can easily find the displacement expressions for any double couple orientation. 


\section{§5. Dip-slip fault with an arbitrary dip angle}

First consider a double couple with moment $M(t)$ in the $\left(x_{1}, x_{2}, x_{3}\right)$ coordinate system (Fig. 6). By the results in the previous section, displacements due to this force systern can be derived from the following expression

$$
\text { ' } \boldsymbol{u}=\operatorname{grad} \Phi+\operatorname{curl} \operatorname{curl}\left(0, \Psi_{2}{ }^{\prime}, \Psi_{3}{ }^{\prime}\right)
$$

with

$$
\left.\begin{array}{rl}
\Phi & =\frac{1}{2 \pi \rho} \frac{\partial^{2} \phi}{\partial x_{2} \partial x_{3}}, \\
\Psi_{2}{ }^{\prime} & =-\frac{1}{4 \pi \rho} \frac{\partial \psi}{\partial x_{3}}, \quad \Psi_{3}{ }^{\prime}=-\frac{1}{4 \pi \rho} \frac{\partial \psi}{\partial x_{2}}
\end{array}\right\}
$$

where

$$
\left.\begin{array}{rl}
\phi & =\frac{1}{R} F\left(t-\frac{R}{v_{p}}\right), \\
\phi & =\frac{1}{R} F\left(t-\frac{R}{v_{s}}\right), \\
F(t) & =\int_{0}^{t} d s^{\prime} \int_{0}^{z^{\prime}} M(s) d s,
\end{array}\right\}
$$

$R$ being the radial distance from the origin.

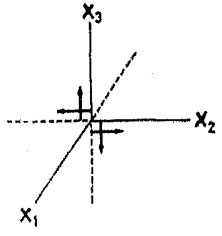

Fig. 6.

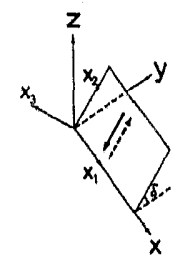

Fig. 7 .
Now, if the $\left(x_{1}, x_{2}, x_{3}\right)$ coordinate system is transformed to the $(x, y, z)$ coordinate system by the relation

$$
\left.\begin{array}{l}
x_{1}=x, \\
x_{2}=y \cos \delta+z \sin \delta, \\
x_{8}=-y \sin \delta+z \cos \delta
\end{array}\right\}
$$

Then, this is equivalent to the dip-slip fault model with a dip angle $\delta$ (Fig. 7). For this coordinate system, the displacement components are given by

$$
\boldsymbol{u}=\operatorname{grad} \Phi+\operatorname{curl} \operatorname{curl}\left(0, \Psi_{2}{ }^{\prime \prime}, \Psi_{3}{ }^{\prime \prime}\right)
$$

where

$$
\begin{aligned}
\Phi= & \frac{1}{2 \pi \rho} \frac{\partial^{2} \phi}{\partial x_{2} \partial x_{3}}=\frac{1}{2 \pi \rho}\left(\cos \delta \frac{\partial}{\partial y}+\sin \delta \frac{\partial}{\partial z}\right) \\
& \times\left(-\sin \delta \frac{\partial}{\partial y}+\cos \delta \frac{\partial}{\partial z}\right) \phi \\
= & -\frac{1}{4 \pi \rho}\left[\sin 2 \delta\left(\frac{\partial^{2}}{\partial y^{2}}-\frac{\partial^{2}}{\partial z^{2}}\right)\right. \\
& \left.-2 \cos 2 \delta \frac{\partial^{2}}{\partial y \partial z}\right] \phi \\
\Psi_{2}^{\prime \prime} & =\Psi_{2}^{\prime} \cos \delta-\Psi_{3}^{\prime} \sin \delta \\
= & \frac{1}{4 \pi \rho}\left(\sin 2 \delta \frac{\partial}{\partial y}-\cos 2 \delta \frac{\partial}{\partial z}\right) \psi \\
\Psi_{3}^{\prime \prime} & =\Psi_{2}^{\prime} \sin \delta+\Psi_{s}^{\prime} \cos \delta \\
= & -\frac{1}{4 \pi \rho}\left(\cos 2 \delta \frac{\partial}{\partial y}+\sin 2 \delta \frac{\partial}{\partial z}\right) \psi
\end{aligned}
$$

Next the Cartesian coordinate system ( $x$, $y, z)$ is transformed to a cylindrical coordinate system $(r, \varphi, z)$ by the relation

$$
x=r \cos \varphi, \quad y=r \sin \varphi, \quad z=z .
$$

Then the displacements are

$$
\boldsymbol{u}=\operatorname{grad} \Phi+\operatorname{curl} \operatorname{curl}\left(\Psi_{1}{ }^{\prime \prime \prime}, \Psi_{2}{ }^{\prime \prime \prime}, \Psi_{\mathbf{8}}{ }^{\prime \prime \prime}\right)
$$

where

$$
\begin{gathered}
\Phi=\frac{1}{4 \pi \rho}\left[-\sin 2 \delta\left\{\sin ^{2} \varphi \frac{\partial^{2}}{\partial r^{2}}+\frac{\cos ^{2} \varphi}{r} \frac{\partial}{\partial r}\right.\right. \\
\left.\left.-\frac{\partial^{2}}{\partial z^{2}}\right\}+2 \cos 2 \delta \sin \varphi \frac{\partial^{2}}{\partial r \partial z}\right] \phi, \\
\Psi_{1}^{\prime \prime \prime}=\frac{1}{4 \pi \rho} \sin \varphi\left[\sin 2 \delta \sin \varphi \frac{\partial}{\partial r}\right. \\
\left.-\cos 2 \delta \frac{\partial}{\partial z}\right] \phi,
\end{gathered}
$$

$$
\begin{aligned}
\Psi_{2}{ }^{\prime \prime \prime}=\frac{1}{4 \pi \rho} \cos \varphi[\sin 2 \delta & \sin \varphi \frac{\partial}{\partial r} \\
& \left.-\cos 2 \delta \frac{\partial}{\partial z}\right] \psi,
\end{aligned}
$$

$\Psi_{s}{ }^{\prime \prime \prime}=-\frac{1}{4 \pi \rho}\left[\cos 2 \delta \sin \varphi \frac{\partial}{\partial r}+\sin 2 \delta \frac{\partial}{\partial z}\right] \psi$.

When $M(t)=M_{0} e^{i \omega t}, \phi$ and $\phi$ can be expressed by

$$
\left.\begin{array}{rl}
\phi & =-\frac{M_{0}}{\omega^{2}} \frac{1}{R} e^{i \omega\left(t-R / v_{p}\right)} \\
& =-\frac{M_{0}}{\omega^{2}} e^{i \omega t} \int_{0}^{\infty} J_{0}(\xi r) e^{-\nu_{\alpha}|z| \frac{\xi d \xi}{\nu \alpha}} \\
\psi & =-\frac{M_{0}}{\omega^{2}} e^{i \omega t} \int_{0}^{\infty} J_{0}(\xi r) e^{-\nu_{\beta}|z| \frac{\xi d \xi}{\nu_{\beta}}}
\end{array}\right\}
$$


with

$$
\nu_{\alpha}=\sqrt{\xi^{2}-h^{2}}, \quad \nu_{\beta}=\sqrt{\xi^{2}-k^{2}}, \quad h=\omega / v_{p}, \quad k=\omega / v_{\mathrm{s}} .
$$

Substitution of (28) into (27) yields

$$
\begin{aligned}
& \Phi=-\frac{M_{0}}{4 \pi \rho \omega^{2}} e^{i \omega t} \int_{0}^{\infty}\left[\frac{1}{2} \sin 2 \delta\left\{\xi^{2} \cos 2 \varphi J_{2}(\xi r)+\left(2 \nu_{\alpha}{ }^{2}+\xi^{2}\right) J_{0}(\xi r)\right\}\right. \\
& \left.+2 \cos 2 \delta \sin \varphi \operatorname{sgn} z \xi \nu_{\alpha} J_{1}(\xi r)\right] \frac{\xi e^{-\nu_{\alpha}|z|}}{\nu_{\alpha}} d \xi, \\
& \left.\Psi_{1}^{\prime \prime \prime}=\frac{M_{0}}{4 \pi \rho \omega^{2}} e^{i \omega t} \sin \varphi \int_{0}^{\infty}\left[\sin 2 \delta \sin \varphi \xi J_{1}(\xi r)-\cos 2 \delta \operatorname{sgn} z \nu_{\beta} J_{0}(\xi r)\right] \frac{\xi e^{-\nu} \beta^{|z|}}{\nu_{\beta}} d \xi,\right\} \\
& \Psi_{2}^{\prime \prime \prime}=\frac{M_{0}}{4 \pi \rho \omega^{2}} e^{i \omega t} \cos \varphi \int_{0}^{\infty}\left[\sin 2 \delta \sin \varphi \xi J_{1}(\xi r)-\cos 2 \delta \operatorname{sgn} z \nu_{\beta} J_{0}(\xi r)\right] \frac{\xi e^{-\nu_{\beta}|x|}}{\nu_{\beta}} d \xi,
\end{aligned}
$$

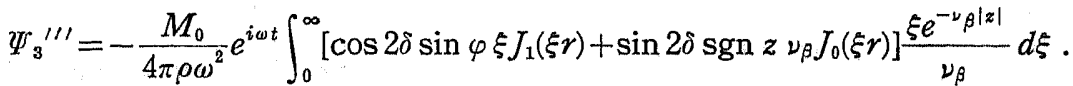

In cylindrical coordinates, the displacements can also be written as

$$
\boldsymbol{u}=\operatorname{grad} \Phi+\operatorname{curl} \operatorname{curl}\left(0,0, \Psi_{V}\right)+\operatorname{curl}\left(0,0, \Psi_{H}\right) .
$$

After some calculations, we can show that

$$
\left.\begin{array}{rl}
\Psi_{V}=-\frac{M_{0}}{4 \pi \rho \omega^{2}} e^{i \omega t} \int_{0}^{\infty} & {\left[\frac{3}{2} \sin 2 \delta \operatorname{sgn} z \nu_{\beta} J_{0}(\xi r)+\cos 2 \delta \sin \varphi \frac{2 \xi^{2}-k^{2}}{\xi} J_{1}(\xi r)\right.} \\
& \left.+\frac{1}{2} \sin 2 \delta \cos 2 \varphi \operatorname{sgn} z \nu_{\beta} J_{2}(\xi r)\right] \frac{\xi e^{-\nu_{\beta}|z|}}{\nu_{\beta}} d \xi, \\
\Psi_{H}=\frac{M_{0}}{4 \pi \rho \omega^{2}} e^{i \omega t} \int_{0}^{\infty}\left[\cos 2 \delta \cos \varphi \frac{\operatorname{sgn} z \nu_{\beta} k^{2}}{\xi} J_{1}(\xi r)-\frac{1}{2} \sin 2 \delta \sin 2 \varphi k^{2} J_{2}(\xi r)\right] \frac{\xi e^{-\nu \beta}|z|}{\nu_{\beta}} d \xi . .
\end{array}\right\}
$$

These expressions are useful when solving problems in a medium with plane boundaries, for example, surface wave propagation in a multi-layered semi-infinite medium.

Finally, in spherical coordinates $(R, \Theta, \varphi)$ with

we have

$$
R=\sqrt{r^{2}+z^{2}}, \quad \tan \theta=r / z, \quad \varphi=\varphi,
$$

where

$$
\boldsymbol{u}=\operatorname{grad} \Phi+\operatorname{curl} \operatorname{curl}\left(\Psi_{1}, \Psi_{2}, \Psi_{3}\right) \text {, }
$$

$$
\begin{aligned}
& \Phi=\frac{1}{4 \pi \rho}\left[\sin 2 \delta\left(\cos ^{2} \Theta-\sin ^{2} \Theta \sin ^{2} \varphi\right)+\cos 2 \delta \sin 2 \Theta \sin \varphi\right] R \frac{d}{d R}\left(\frac{1}{R} \frac{d \phi}{d R}\right), \\
& \Psi_{1}=-\frac{1}{4 \pi \rho}\left[\sin 2 \delta\left(\cos ^{2} \Theta-\sin ^{2} \Theta \sin ^{2} \varphi\right)+\cos 2 \delta \sin 2 \Theta \sin \varphi\right] \frac{d \psi}{d R} \\
& \Psi_{2}=\frac{1}{4 \pi \rho}\left[\sin 2 \delta \frac{\sin 2 \Theta}{2}\left(1+\sin ^{2} \varphi\right)-\cos 2 \delta \cos 2 \Theta \sin \varphi\right] \frac{d \psi}{d R}=\frac{1}{2} \frac{\partial \Psi_{1}}{\partial \Theta} \\
& \Psi_{3}=\frac{1}{4 \pi \rho}[\sin 2 \delta \sin \Theta \sin \varphi-\cos 2 \delta \cos \Theta] \cos \varphi \frac{d \psi}{d R}=\frac{1}{2 \sin \Theta} \frac{\partial \Psi_{1}}{\partial \varphi}
\end{aligned}
$$

Denoting

$$
\Psi^{\prime}=-\frac{1}{2} R \frac{\partial}{\partial R}\left(\frac{\Psi_{1}}{R}\right),
$$

it can be easily verified that
$\operatorname{curl}\left(\Psi_{1}, \frac{1}{2} \frac{\partial \Psi_{1}}{\partial \Theta}, \frac{1}{2 \sin \Theta} \frac{\partial \Psi_{1}}{\partial \varphi}\right)=\operatorname{curl}\left(R \Psi^{\prime}, 0,0\right)$ and therefore (34) can be reduced to

$$
\boldsymbol{u}=\operatorname{grad} \Phi+\operatorname{curl} \operatorname{curl}(R \Psi, 0,0)
$$


with

$$
\left.\begin{array}{l}
\Phi=A(\delta, \Theta, \varphi) R \frac{d}{d R}\left(\frac{1}{R} \frac{d \phi}{d R}\right), \\
\Psi=\frac{1}{2} A(\delta, \Theta, \varphi) R \frac{d}{d R}\left(\frac{1}{R} \frac{d \psi}{d R}\right),
\end{array}\right\}
$$

and

$$
\begin{aligned}
A(\delta, \Theta, \varphi)= & \frac{1}{4 \pi \rho}\left[\sin 2 \delta P_{2}{ }^{0}(\cos \Theta)\right. \\
& +\frac{2}{3} \cos 2 \delta \sin \varphi P_{2}{ }^{1}(\cos \Theta) \\
& \left.+\frac{1}{6} \sin 2 \delta \cos 2 \varphi P_{2}{ }^{2}(\cos \theta)\right],
\end{aligned}
$$

where $P_{n}{ }^{m}(\cos \Theta)$ are the associated Legendre functions, and are given by

$$
\left.\begin{array}{l}
P_{2}{ }^{0}(\cos \Theta)=\frac{1}{4}(3 \cos 2 \Theta+1), \\
P_{2}{ }^{1}(\cos \theta)=\frac{3}{2} \sin 2 \Theta, \\
P_{2}{ }^{2}(\cos \Theta)=\frac{3}{2}(1-\cos 2 \Theta) .
\end{array}\right\}
$$

$A(\delta, \Theta, \varphi)$ does not depend on frequency, which implies that the radiation pattern is the same for any motion of the source.

Thus, the displacements and stresses are explicitly given by

$$
\begin{aligned}
& u_{R}=\frac{\partial \Phi}{\partial R}-R\left[\nabla^{2} \Psi-\frac{1}{R^{2}} \frac{\partial}{\partial R}\left(R^{2} \frac{\partial \Psi}{\partial R}\right)\right], \\
& u_{\theta}=\frac{1}{R} \frac{\partial}{\partial \Theta}\left[\Phi+\frac{\partial}{\partial R}(R \Psi)\right] \text {, } \\
& u_{\varphi}=\frac{1}{R \sin \Theta} \frac{\partial}{\partial \varphi}\left[\Phi+\frac{\partial}{\partial R}(R \Psi)\right] \text {, } \\
& \widehat{R R}=\lambda \nabla^{2} \Phi+2 \mu^{\frac{\partial^{2} \Phi}{\partial R^{2}}}-2 \mu \frac{\partial}{\partial R} \\
& \times\left[R \nabla^{2} \Psi-\frac{1}{R} \frac{\partial}{\partial R}\left(R^{2} \frac{\partial \Psi}{\partial R}\right)\right], \\
& \widehat{R \Theta}=2 \mu \frac{\partial^{2}}{\partial R \partial \Theta}\left(\frac{\Phi}{R}\right)+\mu \frac{\partial}{\partial \Theta} \\
& \times\left[2\left\{\frac{\partial^{2} \Psi}{\partial R^{2}}+\frac{1}{R} \frac{\partial \Psi}{\partial R}-\frac{1}{R^{2}} \Psi^{\}}\right\}-\nabla^{2} \Psi\right], \\
& \widehat{R \varphi}=\frac{2 \mu}{\sin \Theta} \frac{\partial^{2}}{\partial R \partial \varphi}\left(\frac{\Phi}{R}\right)+\frac{\mu}{\sin \theta} \frac{\partial}{\partial \varphi} \\
& \left.\times\left[2\left\{\frac{\partial^{2} \Psi}{\partial R^{2}}+\frac{1}{R} \frac{\partial \Psi}{\partial R}-\frac{1}{R^{2}} \Psi\right\}-\Gamma^{2} \Psi\right] .\right)
\end{aligned}
$$

It holds that

$$
\frac{\partial u_{\theta}}{\partial \varphi}=\frac{\partial}{\partial \Theta}\left(\sin \Theta u_{\varphi}\right), \quad \frac{\partial}{\partial \varphi} \overparen{R \Theta}=\frac{\partial}{\partial \Theta}(\sin \Theta \widehat{R \varphi}) .
$$

$$
\begin{aligned}
& \text { When } M(t)=M_{0} e^{i \omega t}, \\
& \left.\begin{array}{rl}
\Phi & =\frac{i \omega M_{0}}{v_{p}^{3}} A(\delta, \Theta, \varphi) h_{2}^{(2)}\left(\frac{\omega R}{v_{p}}\right) e^{i \omega t} \\
& =\frac{M_{0}}{v_{p}^{2} R} A\left(1-\frac{3 i v_{p}}{\omega R}-\frac{3 v_{p}^{2}}{\omega^{2} R^{2}}\right) e^{i \omega\left(t-R / v_{p}\right)}, \\
\Psi & =\frac{i \omega M_{0}}{2 v_{s}^{3}} A(\delta, \Theta, \varphi) h_{2}^{(2)}\left(\frac{\omega R}{v_{s}}\right) e^{i \omega t} \\
& =\frac{M_{0}}{2 v_{s}{ }^{2} R} A\left(1-\frac{3 i v_{s}}{\omega R}-\frac{3 v_{s}{ }^{2}}{\omega^{2} R^{2}}\right) e^{i \omega\left(t-R / v_{s}\right)}
\end{array}\right\}
\end{aligned}
$$

and from (40) and (41)

$$
\begin{aligned}
u_{R}= & \frac{\partial \Phi}{\partial R}+\frac{6}{R} \Psi \\
\overparen{R R}= & \mu\left[-\left(\frac{\omega^{2}}{v_{s}{ }^{2}}-\frac{8}{R^{2}}\right) \Phi-4 \frac{\partial}{\partial R}\left(\frac{\Phi}{R}\right)\right. \\
& \left.+12 \frac{\partial}{\partial R}\left(\frac{\Psi}{R}\right)\right], \\
\overparen{R \Theta}= & \mu \frac{\partial}{\partial \Theta}\left[2 \frac{\partial}{\partial R}\left(\frac{\Phi}{R}\right)-\left(\frac{\omega^{2}}{v_{s}{ }^{2}}-\frac{8}{R^{2}}\right) \Psi\right. \\
& \left.-2 \frac{\partial}{\partial R}\left(\frac{\Psi}{R}\right)\right] .
\end{aligned}
$$

For large $R$,

$$
\Phi \approx \frac{M_{0}}{v_{p}{ }^{2}} A \frac{1}{R} e^{i \omega\left(t-R / v_{p}\right)}, \quad \Psi \approx \frac{M_{0}}{2 v_{a}{ }^{2}} A \frac{1}{R} e^{i \omega\left(t-R / v_{s}\right)},
$$

hence we have

$$
\begin{aligned}
& u_{R} \approx \frac{\partial \Phi}{\partial R} \approx \frac{\omega M_{0}}{v_{p}^{3}} A \frac{1}{R} e^{i \omega\left(t-R / v_{p}\right)-i(\pi / 2)}, \\
& u_{\Theta} \approx \frac{\partial^{2} \Psi}{\partial R \partial \Theta} \approx \frac{\omega M_{0}}{2 v_{s}^{3}} \frac{\partial A}{\partial \Theta} \frac{1}{R} e^{i \omega\left(t-R / v_{g}\right)-i(\pi / 2)} \text {, } \\
& u_{\varphi} \approx \frac{1}{\sin \theta} \frac{\partial^{2} \Psi}{\partial R \partial \varphi} \\
& \approx \frac{\omega M_{0}}{2 v_{s}^{2}} \frac{1}{\sin \Theta} \frac{\partial A}{\partial \varphi} \frac{1}{R} e^{i \omega\left(t-R_{i} v_{\sharp}\right)-i(\pi / 2)}, \\
& \widehat{R R} \approx-\frac{\rho \omega^{2} M_{0}}{v_{p}^{2}} A \frac{1}{R} e^{i \omega\left(t-R / v_{p}\right)}, \\
& \overparen{R \Theta} \approx-\frac{\rho \omega^{2} M_{0}}{2 v_{s}^{2}} \frac{\partial A}{\partial \Theta} \frac{1}{R} e^{i \omega\left(t-R / v_{s}\right)}, \\
& \left.\widehat{R \varphi} \approx-\frac{\rho \omega^{2} M_{0}}{2 v_{\mathrm{g}}{ }^{2}} \frac{1}{\sin \Theta} \frac{\partial A}{\partial \varphi} \frac{1}{R} e^{i \omega\left(t-R / v_{\mathrm{s}}\right)} .\right)
\end{aligned}
$$

\section{§6. Strike-slip fault with an arbitrary dip angle}

For the double couple, as illustrated in Fig. 8, 
the displacement vector can be expressed by

$$
\boldsymbol{u}=\operatorname{grad} \Phi+\operatorname{curl} \operatorname{curl}\left(\Psi_{1}{ }^{\prime}, 0, \Psi_{3}{ }^{\prime}\right)
$$

with

$$
\left.\begin{array}{l}
\Phi=-\frac{1}{2 \pi \rho} \frac{\partial^{2} \phi}{\partial x_{1} \partial x_{3}}, \\
\Psi_{1}{ }^{\prime}=\frac{1}{4 \pi \rho} \frac{\partial \psi}{\partial x_{3}}, \quad \Psi_{3}{ }^{\prime}=\frac{1}{4 \pi \rho} \frac{\partial \psi}{\partial x_{1}},
\end{array}\right\}
$$

where $\phi$ and $\phi$ are given by $(2)^{\prime}$.

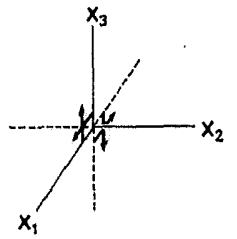

Fig. 8.

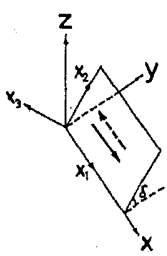

Fig. 9.
The $(x, y, z)$ coordinate system, which is related to the $\left(x_{1}, x_{2}, x_{3}\right)$ system by (22), is used in order to obtain the expressions for a strike-slip fault with a dip angle $\delta$ (Fig. 9). Then we have

$$
\boldsymbol{u}=\operatorname{grad} \Phi+\operatorname{curl} \operatorname{curl}\left(\Psi_{1}^{\prime \prime}, \Psi_{2}^{\prime \prime}, \Psi_{3}^{\prime \prime}\right)
$$

where

$$
\begin{aligned}
& \Phi=-\frac{1}{2 \pi \rho} \frac{\partial}{\partial x}\left(-\sin \delta \frac{\partial}{\partial y}+\cos \delta \frac{\partial}{\partial z}\right) \phi \\
& \Psi_{1}^{\prime \prime}=\frac{1}{4 \pi \rho}\left(-\sin \delta \frac{\partial}{\partial y}+\cos \delta \frac{\partial}{\partial z}\right) \psi, \\
& \Psi_{2}{ }^{\prime \prime}=-\frac{1}{4 \pi \rho} \sin \delta \frac{\partial \psi}{\partial x} \\
& \Psi_{3}{ }^{\prime \prime}=\frac{1}{4 \pi \rho} \cos \delta \frac{\partial \psi}{\partial x} .
\end{aligned}
$$

Again transforming from Cartesian coordinates to cylindrical coordinates, we find

$$
\left.\begin{array}{c}
\boldsymbol{u}=\operatorname{grad} \Phi+\operatorname{curl} \operatorname{curl}\left(\Psi_{1}^{\prime \prime \prime}, \Psi_{2}{ }^{\prime \prime \prime}, \Psi_{3}{ }^{\prime \prime \prime}\right) \\
\Phi=\frac{1}{4 \pi \rho}\left[\sin \delta \sin 2 \varphi\left(\frac{\partial^{2}}{\partial r^{2}}-\frac{1}{r} \frac{\partial}{\partial r}\right)\right. \\
\left.-2 \cos \delta \cos \varphi \frac{\partial^{2}}{\partial r \partial z}\right] \phi, \\
\Psi_{1}^{\prime \prime \prime \prime}=\frac{1}{4 \pi \rho}\left[-\sin \delta \sin 2 \varphi \frac{\partial}{\partial r}\right. \\
\left.+\cos \delta \cos \varphi \frac{\partial}{\partial z}\right] \psi, \\
\Psi_{2}{ }^{\prime \prime \prime}=-\frac{1}{4 \pi \rho}\left[\sin \delta \cos 2 \varphi \frac{\partial}{\partial r}\right. \\
\left.+\cos \delta \sin \varphi \frac{\partial}{\partial z}\right] \psi, \\
\Psi_{3}^{\prime \prime \prime}=\frac{1}{4 \pi \rho} \cos \delta \cos \varphi \frac{\partial \psi}{\partial r} .
\end{array}\right\}
$$

When $M(t)=M_{0} e^{i \omega t}$, we have

$$
\begin{aligned}
& \Phi=-\frac{M_{0}}{4 \pi \rho \omega^{2}} e^{i \omega t} \int_{0}^{\infty}\left[\sin \delta \sin 2 \varphi \xi^{2} J_{2}(\xi r)-2 \cos \delta \cos \varphi \operatorname{sgn} z \xi \nu_{\alpha} J_{1}(\xi r)\right] \frac{\xi e^{-\nu_{\alpha}|z|}}{\nu_{\alpha}} d \xi, \\
& \Psi_{1}^{\prime \prime \prime}=-\frac{M_{0}}{4 \pi \rho \omega^{2}} e^{i \omega t} \int_{0}^{\infty}\left[\sin \delta \sin 2 \varphi \xi J_{1}(\xi r)-\cos \delta \cos \varphi \operatorname{sgn} z \nu_{\beta} J_{0}(\xi r)\right]^{\xi e^{-\nu_{\beta}|z|}} \frac{\nu_{\beta}}{\nu_{\beta}} d \xi \text {, } \\
& \Psi_{2}{ }^{\prime \prime \prime}=-\frac{M_{0}}{4 \pi \rho \omega^{2}} e^{i \omega t} \int_{0}^{\infty}\left[\sin \delta \cos 2 \varphi \xi J_{1}(\xi r)+\cos \delta \sin \varphi \operatorname{sgn} z \nu_{\beta} J_{0}(\xi r)\right]^{\xi e^{-\nu \beta|z|}} \frac{\nu_{\beta}}{\nu_{0}} d \xi \text {, } \\
& \Psi_{3}^{\prime \prime \prime}=\frac{M_{0}}{4 \pi \rho \omega^{2}} e^{i \omega t} \int_{0}^{\infty} \cos \delta \cos \varphi \xi J_{1}(\xi r) \frac{\xi e^{-\nu \beta}|z|}{\nu_{\beta}} d \xi \text {. }
\end{aligned}
$$

If we use the relation (31),

$$
\left.\begin{array}{l}
\Psi_{V}=\frac{M_{0}}{4 \pi \rho \omega^{2}} e^{i \omega t} \int_{0}^{\infty}\left[\cos \delta \cos \varphi \frac{2 \xi^{2}-k^{2}}{\xi} J_{1}(\xi r)-\sin \delta \sin 2 \varphi \operatorname{sgn} z \nu_{\beta} J_{2}(\xi r)\right] \frac{\xi e^{-\nu \beta}|z|}{\nu_{\beta}} d \xi, \\
\Psi_{H}=\frac{M_{0}}{4 \pi \rho \omega^{2}} e^{i \omega t} \int_{0}^{\infty}\left[\cos \delta \sin \varphi \frac{\operatorname{sgn} z \nu_{\beta} k^{2}}{\xi} J_{1}(\xi r)+\sin \delta \cos 2 \varphi k^{2} J_{2}(\xi r)\right] \frac{\xi e^{-\nu \beta|z|}}{\nu_{\beta}} d \xi .
\end{array}\right\}
$$

For the spherical coordinates $(R, \Theta, \varphi)$, we can easily obtain

$$
\boldsymbol{u}=\operatorname{grad} \Phi+\operatorname{curl} \operatorname{curl}\left(\Psi_{1}, \Psi_{2}, \Psi_{3}\right),
$$


where

$$
\left.\begin{array}{l}
\Phi=\frac{1}{4 \pi \rho}\left(\sin \delta \sin ^{2} \Theta \sin 2 \varphi-\cos \delta \sin 2 \Theta \cos \varphi\right) R \frac{d}{d R}\left(\frac{1}{R} \frac{d \phi}{d R}\right), \\
\Psi_{1}=-\frac{1}{4 \pi \rho}\left(\sin \delta \sin ^{2} \Theta \sin 2 \varphi-\cos \delta \sin 2 \Theta \cos \varphi\right) \frac{d \psi}{d R}, \\
\Psi_{2}=-\frac{1}{4 \pi \rho}\left(\sin \delta \frac{\sin 2 \Theta}{2} \sin 2 \varphi-\cos \delta \cos 2 \Theta \cos \varphi\right) \frac{d \psi}{d R}=\frac{1}{2} \frac{\partial \Psi_{1}}{\partial \Theta}, \\
\Psi_{3}=-\frac{1}{4 \pi \rho}(\sin \delta \sin \Theta \cos 2 \varphi+\cos \delta \cos \Theta \sin \varphi) \frac{d \psi}{d R}=\frac{1}{2 \sin \Theta} \frac{\partial \Psi_{1}}{\partial \varphi} .
\end{array}\right\}
$$

Similarly to the dip-slip fault model, we finally obtain the same expressions as in (37) through (46), taking $B(\delta, \Theta, \varphi)$ in place of $A(\delta, \Theta, \varphi)$, where $B(\delta, \Theta, \varphi)=\frac{1}{4 \pi \rho}\left[-\frac{2}{3} \cos \delta \cos \varphi P_{2}{ }^{1}(\cos \Theta)\right.$

$$
\left.+\frac{1}{3} \sin \delta \sin 2 \varphi P_{2}{ }^{2}(\cos \theta)\right] \text {. }
$$

$\S 7$. Expressions for permanent displacements and stresses

Applying an operator

$$
\frac{1}{2 \pi i} \int_{-i \varepsilon-\infty}^{-i \varepsilon+\infty} \frac{d \omega}{\omega}
$$

to the expressions in (40) and (41), and taking $\Phi$ and $\Psi$ given by (43), we have responses when the original movement of the double couple is a step function. Taking into account of the following relations

$$
\begin{aligned}
\frac{1}{2 \pi i} \int_{-i \varepsilon \cdots \infty}^{-i a+\infty} \frac{1}{\omega} \Phi d \omega=- & \frac{M_{0}}{2 v_{p}^{2} R} A\left[1-\frac{3 v_{p}^{2}}{R^{2}} t^{2}\right] H\left(\tau_{p}\right), \\
\frac{1}{2 \pi i} \int_{-i \varepsilon-\infty}^{-i c+\infty} \frac{1}{\omega} \frac{\partial \Phi}{\partial R} d \omega= & -\frac{M_{0}}{v_{p}^{3} R} A \delta\left(\tau_{p}\right)+\frac{M_{0}}{2 v_{p}^{2} R^{2}} \\
& \times A\left[1-\frac{9 v_{p}^{2}}{R^{2}} t^{2}\right] H\left(\tau_{p}\right), \\
\frac{1}{2 \pi i} \int_{-i \varepsilon-\infty}^{-i \varepsilon+\infty} \frac{1}{\omega} \omega^{2} \Phi d \omega= & -\frac{M_{0}}{v_{p}^{2} R} A\left[\delta^{\prime}\left(\tau_{p}\right)+\frac{3 v_{p}}{R} \delta\left(\tau_{p}\right)\right. \\
& \left.+\frac{3 v_{p}^{2}}{R^{2}} H\left(\tau_{p}\right)\right],
\end{aligned}
$$

where $\tau_{p, s}=t-R / v_{p, s}, H(\tau)$ is the step function, $\delta(\tau)$ is the delta function and $\delta^{\prime}(\tau)=\partial \delta(\tau) / \partial t$, then we can find

$$
\left.\begin{array}{rl}
u_{R}= & -\frac{M_{0}}{v_{p}{ }^{3} R} A \delta\left(\tau_{p}\right)+\frac{M_{0}}{2 v_{s}{ }^{2} R^{2}} A\left\{\frac{v_{s}{ }^{2}}{v_{p}^{2}} H\left(\tau_{p}\right)\right. \\
& \left.-3 H\left(\tau_{s}\right)\right\}-\frac{9 M_{0}}{2 R^{4}} A t^{2}\left\{H\left(\tau_{p}\right)-H\left(\tau_{s}\right)\right\}, \\
u_{\theta}= & \frac{1}{R} \frac{\partial U}{\partial \Theta}, \quad u_{\varphi}=\frac{1}{R \sin \Theta} \frac{\partial U}{\partial \varphi},
\end{array}\right\}
$$

with

$$
\begin{aligned}
U= & -\frac{M_{0}}{2 v_{s}^{3}} A \delta\left(\tau_{s}\right)-\frac{M_{0}}{2 v_{p}^{2} R} A H\left(\tau_{p}\right) \\
& +\frac{3 M_{0}}{2 R^{3}} A t^{2}\left\{H\left(\tau_{p}\right)-H\left(\tau_{s}\right)\right\}
\end{aligned}
$$

and

$$
\begin{aligned}
\frac{\overparen{R R}}{\mu M_{0}}= & \frac{1}{v_{p}^{2} v_{s}^{2} R} A \delta^{\prime}\left(\tau_{p}\right)+\frac{1}{v_{s}^{9} R} \\
& \times A\left\{\frac{v_{s}}{v_{p}}\left(3+4 \frac{v_{s}{ }^{2}}{v_{p}{ }^{2}}\right) \delta\left(\tau_{p}\right)-6 \delta\left(\tau_{s}\right)\right\} \\
& +\frac{1}{v_{s}^{2} R^{3}} A\left\{\left(3-8 \frac{v_{s}{ }^{2}}{v_{p}{ }^{2}}\right) H\left(\tau_{p}\right)+6 H\left(\tau_{s}\right)\right\} \\
& +\frac{36}{R^{5}} A t^{2}\left\{H\left(\tau_{p}\right)-H\left(\tau_{s}\right)\right\} \\
\frac{\overparen{R \Theta}}{\mu M_{0}}= & \frac{\partial S}{\partial \Theta}, \quad \frac{\overparen{R \varphi}}{\mu M_{0}}=\frac{1}{\sin \Theta} \frac{\partial S}{\partial \varphi},
\end{aligned}
$$

with

$$
\begin{aligned}
S= & \frac{1}{2 v_{s}{ }^{4} R} A \delta^{\prime}\left(\tau_{s}\right)+\frac{1}{v_{s}^{3} R^{2}} A\left\{-2 \frac{v_{s}^{3}}{v_{p}^{8}} \delta\left(\tau_{p}\right)+\frac{5}{2} \delta\left(\tau_{s}\right)\right\} \\
& +\frac{1}{v_{s}^{2} R^{3}} A\left\{2 \frac{v_{s}{ }^{2}}{v_{p}^{2}} H\left(\tau_{p}\right)-3 H\left(\tau_{s}\right)\right\} \\
& -\frac{12}{R^{5}} A t^{2}\left\{H\left(\tau_{p}\right)-H\left(\tau_{s}\right)\right\} .
\end{aligned}
$$

Therefore the permanent displacements and stresses are

$$
\left.\begin{array}{l}
u_{R}=-\frac{M_{0}}{2 v_{s}^{2} R^{2}} \frac{3 \lambda+5 \mu}{\lambda+2 \mu} A, \\
u_{\theta}=-\frac{M_{0}}{2 v_{s}^{2} R^{2}} \frac{\mu}{\lambda+2 \mu} \frac{\partial A}{\partial \Theta}, \\
u_{\varphi}=-\frac{M_{0}}{2 v_{s}^{2} R^{2}} \frac{\mu}{\lambda+2 \mu} \frac{1}{\sin \Theta} \frac{\partial A}{\partial \varphi},
\end{array}\right\}
$$




$$
\left.\begin{array}{l}
\widehat{R R}=\frac{\mu M_{0}}{v_{s}{ }^{3}} \frac{9 \lambda+10 \mu}{\lambda+2 \mu} A, \\
\widehat{R \Theta}=-\frac{\mu M_{0}}{v_{s}^{2} R^{3}} \frac{3 \lambda+4 \mu}{\lambda+2 \mu} \frac{\partial A}{\partial \Theta}, \\
\overparen{R \varphi}=-\frac{\mu M_{0}}{v_{s}{ }^{3} R^{3}} \frac{3 \lambda+4 \mu}{\lambda+2 \mu} \frac{1}{\sin \Theta} \frac{\partial A}{\partial \varphi},
\end{array}\right\}
$$

where $\lambda$ and $\mu$ are Lamé's constants.

For the strike-slip fault model, only the replacement of $A(\delta, \Theta, \varphi)$ by $B(\delta, \Theta, \varphi)$ is required.

\section{§8. Moving dislocation case}

Take a point whose ordinates are $\left(\xi_{1}, \xi_{2}\right)$ on the fault plane $\left(x_{1} x_{2}\right.$-plane in Fig. 7 or Fig. 9). Assuming $x_{1^{-}}$and $x_{2}$-components of the rupture velocity to be $c_{1}$ and $c_{2}$, respectively, the disturbance due to a dislocation propagated from $x_{1}=0$ to $x_{1}=L$ and from $x_{2}=0$ to $x_{2}=w$ with their respective velocities is given by taking $M_{0}$ in the previous sections as an operator

$\mu \int_{\xi_{2}=0}^{w} \int_{\xi_{1}=0}^{L} d \xi_{1} d \xi_{2} U_{0}\left(\xi_{1}, \xi_{2} ; \omega\right) e^{\left.\left.-i \mid \omega / c_{1}\right) \hat{\xi}_{1}-i \mid \omega / c_{2}\right) \xi_{2}}$

where $U_{0}$ is the Fourier transform of the dislocation $U_{0}^{*}(t)$ with respect to $t$, that is,

$$
U_{0}(\omega)=\int_{-\infty}^{\infty} U_{0}^{*}(t) e^{-i \omega t} d t
$$

and $R$ should be taken as

$$
R \rightarrow R-\frac{x_{1}}{R} \xi_{1}-\frac{x_{2}}{R} \xi_{2},
$$

assuming that $L / R \ll 1$ and $w / R \ll 1$. Then $\phi$ is found

$$
\begin{aligned}
\phi= & -\frac{\mu}{\omega^{2} R} e^{i \omega\left(t-R / v_{p}\right)} \int_{0}^{w} \int_{0}^{L} U_{0}\left(\xi_{1}, \xi_{2} ; \omega\right) \\
& \times e^{-i 2 X_{p}\left(\xi_{1} / L\right)-i 2 Y_{p}\left(\xi_{2} / w\right)} d \xi_{1} d \xi_{2}
\end{aligned}
$$

with

$$
\left.\begin{array}{l}
X_{p}=\frac{\omega L}{2 v_{p}}\left(\frac{v_{p}}{c_{1}}-\frac{x_{1}}{R}\right), \\
Y_{p}=\frac{\omega w}{2 v_{p}}\left(\frac{v_{p}}{c_{2}}-\frac{x_{2}}{R}\right) .
\end{array}\right\}
$$

If we denote the Fourier spectrum of the mean dislocation over the fault plane by $\bar{U}_{0}$, we have

$$
\phi=-\frac{\mu \bar{U}_{0} S}{\omega^{2}} \frac{\sin X_{p}}{X_{p}} \frac{\sin Y_{p}}{Y_{p}} \frac{1}{R} e^{i \omega\left(t-R / v_{p}\right)-i X_{p}-i Y_{p}} .
$$

$S$ being the area of the fault plane.

In various coordinate systems treated in the previous sections, $X_{p}$ and $Y_{p}$ can be expressed by

$$
\left.\begin{array}{rl}
X_{p} & =\frac{\omega L}{2 v_{p}}\left(\frac{v_{p}}{c_{1}}-\frac{x}{R}\right)=\frac{\omega L}{2 v_{p}}\left(\frac{v_{p}}{c_{1}}-\frac{r}{R} \cos \varphi\right) \\
& =\frac{\omega L}{2 v_{p}}\left(\frac{v_{p}}{c_{1}}-\sin \Theta \cos \varphi\right), \\
Y_{p} & =\frac{\omega w}{2 v_{p}}\left(\frac{v_{p}}{c_{1}}-\frac{y \cos \delta+z \sin \delta}{R}\right) \\
& =\frac{\omega w}{2 v_{p}}\left(\frac{v_{p}}{c_{1}}-\sin \Theta \sin \varphi \cos \delta\right. \\
& -\cos \Theta \sin \delta) .
\end{array}\right\}
$$

Similarly to $\phi$, we have

$$
\begin{aligned}
\psi= & -\frac{\mu \bar{U}_{0} S}{\omega^{2}} \frac{\sin X_{8}}{X_{\mathrm{s}}} \frac{\sin Y_{\mathrm{s}}}{Y_{\mathrm{s}}} \frac{1}{R} \\
& \times e^{i \omega\left|t-R / v_{\mathrm{s}}\right|-i X_{\mathrm{s}}-i Y_{\mathrm{s}}},
\end{aligned}
$$

where $X_{s}$ and $Y_{s}$ can be given by the same expressions as in (69) by taking $v_{s}$ in place of $v_{p}$.

From these results, it may be understood that the solution for the moving dislocation model can be obtained by replacing $M_{0}$ in the previous sections by

$$
\mu \bar{U}_{0} S \frac{\sin X_{p, s}}{X_{p, s}} \frac{\sin Y_{p, 8}}{Y_{p, s}} e^{-i X_{p, 8}-i Y_{p, s}}
$$

to the first approximation.

In the cylindrical coordinates when the displacement potentials are given by integral expressions as in (32) or (54), the same operator as (64) should be applied after evaluating the integrals. For this case, $r$ and $z$ should be understood as

$r-\xi_{1} \cos \varphi-\xi_{2} \cos \delta \sin \varphi$ and $z-\xi_{2} \sin \delta$ respectively.

\section{\$9. Translation of origin of polar coordinates}

When $M(t)=M_{0} e^{i \omega t}$, results in the polar 
coordinates obtained in $\S 5$ and $\$ 6$ are sum- with marized as follows:

Defining $\phi_{n+m, m}$ and $\phi_{n+m, m}$ by

$\phi_{n+m, m}(R, \Theta, \varphi)=\frac{i \omega M_{0}}{4 \pi \rho v_{p}^{3}} h_{n+m}^{(2)}(h R) P_{n+m}^{m}(\cos \Theta)$ $\times\left(\begin{array}{c}\alpha_{m} \cos m \varphi \\ \beta_{m} \sin m \varphi\end{array}\right) e^{i \omega t}$

$\psi_{n+m, m}(R, \Theta, \varphi)=\frac{i \omega M_{0}}{8 \pi \rho v_{s}{ }^{3}} h_{n+m}^{(2)}(k R) P_{n+m}^{m}(\cos \Theta)$ $\times\left(\begin{array}{c}\alpha_{m} \cos m \varphi \\ \beta_{m} \sin m \varphi\end{array}\right) e^{i \omega t}$

we have

$$
\Phi=\sum_{m=0}^{2} \phi_{2, m}, \quad \Psi=\sum_{m=0}^{2} \phi_{2, m}
$$

where

$$
\left.\begin{array}{lll}
\alpha_{0}=\sin 2 \delta, & \alpha_{1}=0, & \alpha_{2}=\frac{1}{6} \sin 2 \delta \\
\beta_{0}=0, & \beta_{1}=\frac{2}{3} \cos 2 \delta, & \beta_{2}=0,
\end{array}\right\}
$$

for the dip-slip fault model,

$$
\left.\begin{array}{lll}
\alpha_{0}=0, & \alpha_{1}=-\frac{2}{3} \cos \delta, & \alpha_{2}=0, \\
\beta_{0}=0, & \beta_{1}=0, & \beta_{2}=\frac{1}{3} \sin \delta
\end{array}\right\}
$$

for the strike-slip fault model, and

$$
h=\omega / v_{p}, \quad k=\omega / v_{s} .
$$

Then the displacement vector in the polar coordinates $(R, \Theta, \varphi)$ is given by

$$
\boldsymbol{u}=\sum_{m=0}^{2} \boldsymbol{u}_{m}
$$

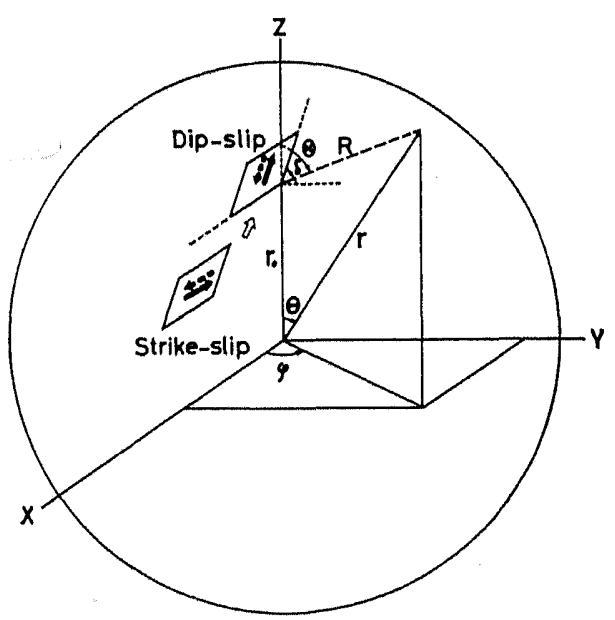

Fig. 10.

$$
\boldsymbol{u}_{m}=\operatorname{grad} \phi_{2, m}+\operatorname{curl} \operatorname{curl}\left(R \psi_{2, m}, 0,0\right) \text {. }
$$

Now the problem presented here is to obtain the displacement vector in another polar coordinates $(r, \theta, \varphi)$, as shown in Fig. 10.

In the $(r, \theta, \varphi)$ system, we can easily find

$$
\begin{aligned}
\boldsymbol{u}_{m}= & \operatorname{grad} \phi_{2, m} \\
& +\operatorname{curl} \operatorname{curl}\left(\left(r-r_{0} \cos \theta\right) \psi_{2, m}, r_{0} \sin \theta \psi_{2, m}, 0\right) \\
= & \operatorname{grad} \phi_{2, m} \\
& +\operatorname{curl} \operatorname{curl}\left(r \psi_{2, m}, 0,0\right) \\
& +\operatorname{curl} \operatorname{curl}\left(-r_{0} \cos \theta \psi_{2, m}, r_{0} \sin \theta \phi_{2, m}, 0\right) .
\end{aligned}
$$

In general, according to Usami et al. (1962), we have an identity

$$
\begin{aligned}
& \operatorname{curl} \operatorname{curl}\left(-r_{0} \cos \theta \phi_{n+m, m}, r_{0} \sin \theta \phi_{n+m, m}, 0\right) \\
&=-\frac{(n+1) k r_{0}}{(n+m+1)(2 n+2 m+1)} \\
& \times \operatorname{curl} \operatorname{curl}\left(r \phi_{n+m+1, m}, 0,0\right) \\
&-\frac{(n+2 m) k r_{0}}{(n+m)(2 n+2 m+1)} \\
& \times \operatorname{curl} \operatorname{curl}\left(r \phi_{n+m-1, m}, 0,0\right) \\
&-\frac{k^{2} r_{0}}{(n+m)(n+m+1)} \frac{\partial}{\partial \varphi} \operatorname{curl}\left(r \phi_{n+m, m}, 0,0\right),
\end{aligned}
$$

whence

$$
\begin{aligned}
\boldsymbol{u}_{m}= & \operatorname{grad} \phi_{2, m}+\operatorname{curl} \operatorname{curl}\left(r \psi_{2, m}, 0,0\right) \\
& -\frac{3-m}{15} k r_{0} \operatorname{curl} \operatorname{curl}\left(r \psi_{3, m}, 0,0\right) \\
& -\frac{2+m}{10} k r_{0} \operatorname{curl} \operatorname{curl}\left(r \phi_{1, m}, 0,0\right) \\
& -\frac{1}{6} k^{2} r_{0} \frac{\partial}{\partial \varphi} \operatorname{curl}\left(r \psi_{2, m}, 0,0\right) .
\end{aligned}
$$

$\phi_{2, m}$ and $\phi_{2, m}$ are functions of $R, \Theta$, and $\varphi$. Onda and Sato (1969) showed that

$$
\begin{aligned}
\frac{\phi_{n+m, m}(R, \Theta, \varphi)}{\left(i \omega M_{0} / 4 \pi \rho v_{p}^{3}\right)}= & \sum_{l=0}^{\infty} \varepsilon_{l}^{n m}\left(h r_{0}\right) j_{l+m}(h r) P_{l+m}^{m}(\cos \theta) \\
& \times\left(\begin{array}{c}
\alpha_{m} \cos m \varphi \\
\beta_{m} \sin m \varphi
\end{array}\right) e^{i \omega t}, \quad r_{0}>r, \\
= & \sum_{l=0}^{\infty} \zeta_{l}^{n m}\left(h r_{0}\right) h_{l+m}^{(2)}(h r) P_{l+m}^{m}(\cos \theta) \\
& \times\left(\begin{array}{c}
\alpha_{m} \cos m \varphi \\
\beta_{m} \sin m \varphi
\end{array}\right) e^{i \omega t}, \quad r>r_{0},
\end{aligned}
$$


where

$$
\left.\begin{array}{rl}
\varepsilon_{l}^{n m}(x)= & (2 m-1) ! !(2 m+2 l+1) i^{n} \\
& \times C_{n}^{m+1 / 2}(-i d / d x) h_{l+m}^{(2)}(x) / x^{m} \\
\zeta_{l}^{n m}(x)= & (2 m-1) ! !(2 m+2 l+1) i^{n} \\
& \times C_{n}^{m+1 / 2}(-i d / d x) j_{l+m}(x) / x^{m}, \\
C_{n}^{m+1 / 2}(x)= & \frac{2^{m} m !}{(2 m) !\left(1-x^{2}\right)^{m / 2}} P_{n+m}^{m}(x) \\
& (\text { Gegenbauer polynomials) } .
\end{array}\right\}
$$

In the present problem, we need only the cases when $n=0,1,2$, and 3 . For these cases,

$$
\left.\begin{array}{rl}
\varepsilon_{l}^{0 m}(x)= & (2 m-1) ! !(2 m+2 l+1) h_{l+m}^{(2)}(x) / x^{m}, \\
\varepsilon_{l}^{1 m}(x)= & (2 m+1) ! !(2 m+2 l+1) \\
& \times\left\{l h_{l+m}^{(2)}(x) / x^{m+1}-h_{l+m+1}^{(2)}(x) / x^{m}\right\}, \\
\varepsilon_{l}^{2 m}(x)= & (2 m+3) ! !(2 m+2 l+1)\left[\frac{l(l-1)}{2} \frac{h_{l+m}^{(2)}(x)}{x^{m+2}}\right. \\
& \left.+(m+1)\left\{\frac{h_{l+m+1}^{(2)}(x)}{x^{m+1}}-\frac{h_{l+m}^{(2)}(x)}{(2 m+3) x^{m}}\right\}\right], \\
\varepsilon_{l}^{30}(x)= & (2 l+1)\left[\left\{1-\frac{5}{2 x^{2}}\left(l^{2}+l+6\right)\right\} h_{l+1}^{(2)}(x)\right. \\
& \left.-\frac{1}{x}\left\{(l-5)-\frac{5}{2 x^{2}} l(l-1)(l-2)\right\} h_{l}^{(2)}(x)\right],
\end{array}\right\}
$$

and $\zeta_{l}^{n m}(x)$ can be obtained by replacing $h_{l+m}^{(2)}(x)$ by $j_{l+m}(x) . \psi_{n+m, m}(R, \Theta, \varphi) /\left(i \omega M_{0} / 8 \pi \rho v_{s}{ }^{3}\right)$ is simi- larly expressed by taking $k$ in place of $h$.

Thus the problem is completely solved. These expressions are useful when solving problems of generation of seismic waves and excitation of the free oscillations due to a dislocation in a spherical earth. Application of these expressions will be given in a future publication.

\section{§9. Acknowledgments}

The author is indebted to Dr. A. F. Espinosa who kindly reviewed the manuscript and offered valuable advices.

\section{References}

Keilis-Borok, V. I., Concerning the Determination of the Dynamic Parameters of a Focus, Trudy Geofiz. In-ta AN SSSR, No. 9, 1950.

Love, A. E. H., A Treatise on the Mathematical Theory of Elasticity, 4th ed., 304-305, Cambridge, University Press, 1927.

Onda, I. and R. Sato, Transformation of an Elastic Wave Solution related to Translation of the Origin of the Polar Coordinate System, Bull. Earthq. Res. Inst., 47, 599-611, 1969.

Usami, T., K. Kano and Y. Satô, Some Remarks on the Solutions of the Equation of Motion in an Isotropic and Homogeneous Elastic Body (II), Zisin, II, 15, 261-269, 1962.

(Received May 10, 1969) 